\title{
Determinants of Dental Care Attendance during Pregnancy: A Systematic Review
}

\author{
Juliana Schaia Rocha ${ }^{a}$ b Letícia Yumi Arima $^{b}$ Renata lani Werneck ${ }^{b}$ \\ Samuel Jorge Moysés ${ }^{b}$ Márcia Helena Baldani ${ }^{a}$ \\ a State University of Ponta Grossa, Ponta Grossa, and b Pontifical Catholic University of Paraná, Curitiba, Brazil
}

\section{Keywords}

Dental care · Dental health services · Oral health .

Pregnancy $\cdot$ Review

\begin{abstract}
Despite the fact that dental care attendance during pregnancy has been recommended by guidelines and institutions, the demand for dental services is still low among pregnant women. The aim of this study was to identify and analyze the determinants of dental care attendance during pregnancy. We performed a systematic literature search in the electronic databases PubMed, Scopus, Web of Science, Latin American and Caribbean Health Sciences Literature, Brazilian Library in Dentistry, Cumulative Index to Nursing and Allied Health Literature, and Medline using relevant keywords. Studies were filtered by publication year (2000-2016) and language (English, Portuguese, Spanish, and French). The included studies were assessed for quality. Their characteristics and statistically significant factors were reported. Fourteen papers were included in the review. The prevalence of dental service usage during pregnancy ranged from 16 to $83 \%$. Demographic factors included women's age, marital status, parity, and nationality. The socioeconomic
\end{abstract}

factors were income, educational level, and type of health insurance. Many psychological and behavioral factors played a role, including oral health practices, oral health and pregnancy beliefs, and health care maintenance. Referred symptoms of gingivitis, dental pain, or dental problems were perceived need. Demographic, socioeconomic, psychological, behavioral factors and perceived need were associated with the utilization of dental services during pregnancy. More well-designed studies with reliable outcomes are required to confirm the framework described in this review.

(c) 2018 S. Karger AG, Basel

The pattern of dental service utilization is a worldwide public health challenge that has been found to be a key predictor of oral health outcomes [Petersen, 2008]. Several studies have identified the determinants of dental service utilization among adults around the world. Some of them are age, sex, race, ethnicity, cost of dental treatment, health insurance, dental anxiety, hygiene habits, oral health education, oral health beliefs, self-reported oral health problems, and need for oral health [Rosenstock, 1966; Scheutz and Heidmann, 2001; Pohjola et al., 2007; Slack-Smith et al., 2007; Araújo et al., 2009; Baker,

\section{KARGER}

(C) 2018 S. Karger AG, Basel

E-Mail karger@karger.com

www.karger.com/cre
Juliana Schaia Rocha

State University of Ponta Grossa

R. Alferes Ângelo Sampaio

Curitiba, PR 80730-460 (Brazil)

E-Mail julianaschaia@ hotmail.com 
2009; Locker et al., 2011; Pinto et al., 2012; Hill et al., 2013]. The knowledge about the nature of these associations can lead to a better understanding of the dental service utilization pattern and may direct the formulation of effective interventions to improve access to dental services [Reisine, 1987; Marshman et al., 2012].

In regard to vulnerable groups, pregnant women included, this assessment is even more important because individuals belonging to such groups are more susceptible to negative health outcomes [Aday, 1994; Woods et al., 2005; National Research Council, 2012]. In addition to the access barriers that adults usually face, there are some barriers that are inherent to this life cycle, such as patients' and health professionals' beliefs regarding dental treatment security and possible changes in oral health status [Dinas et al., 2007; Le et al., 2009; Detman et al., 2010].

While it is generally agreed that oral diseases can result in adverse consequences for the woman in her pregnancy process, there is some controversy as to whether such diseases may result in adverse outcomes for the fetus and the newborn. Systematic reviews demonstrate that periodontal disease during pregnancy may be a potential risk factor for prematurity, low birth weight, and other negative pregnancy outcomes. However, the relationships remain inconclusive, as some associations should be very low with proper corrections of biased study methodologies and heterogeneity [Santos Neto et al., 2012; Shanthi et al., 2012; Ide and Papapanou, 2013; Corbella et al., 2016; Kumar, 2016].

Pregnant women's oral health may worsen due to hormones or changes in diet and hygiene; it may even affect their quality of life in many different ways: e.g., as oral pain, psychological discomfort, physical and psychological disability, social disability, and handicap [Oliveira and Nadanovsky, 2006; Lu et al., 2015]. Periodontal diseases are accentuated because of hormonal changes: higher rates of gingival inflammation have been observed in pregnant women compared to nonpregnant ones [Figuero et al., 2013]. Furthermore, some studies have shown a high prevalence of tooth decay among pregnant women [Radnai et al., 2007; Vergnes et al., 2013]. This may be related to some behavioral changes that can occur during pregnancy, such as difficulties to performing good oral hygiene and increased sugar consumption, leading to higher bacterial plaque scores [Vergnes et al., 2013; Amin and ElSalhy, 2014].

To preserve oral health during pregnancy, a healthy diet and proper oral hygiene are essential [Jevtić et al., 2015]. However, regardless of all possible adverse out- comes and the importance of seeking preventive care in this condition, studies have shown rates as high as 56$74 \%$ in pregnant women who have reported no dental visits [Boggess et al., 2010; Marchi et al., 2010; Vergnes et al., 2013]. Well-designed prenatal care policies are supposed to strengthen and encourage dental service utilization and should aim to have a positive impact on the attitudes and beliefs regarding oral health.

Despite policy efforts aimed at the main cultural or contextual barriers in several countries [Albuquerque et al., 2004; Anderson et al., 2009-2010; Keirse and Plutzer, 2010; Thompson et al., 2013; NHS, 2016], oral health care during pregnancy still reaches few women, and socioeconomic status remains one of the most important determinants for access to health services [Corchuelo-Ojeda, 2013; Amin and ElSalhy, 2014]. During pregnancy, the impact of the social gradient on the use of dental services even increases besides the inherent factors that determine unequal access to health services by population groups [Andersen, 1968; Aday and Andersen, 1974; Hausmann-Muela et al., 2003; Shaw et al., 2008]. There are several beliefs and myths about dental care during pregnancy; these may include dentists', doctors', or family's resistance, low perceived need, and devaluation of oral health [Dinas et al., 2007; Codato et al., 2008; Le et al., 2009].

Considering the importance of prenatal oral health care, studying the factors that enable or inhibit the use of dental services by pregnant women is crucial for promoting effective public policies. Therefore, this systematic review aimed to identify and analyze the determinants of dental care attendance during pregnancy.

\section{Materials and Methods}

\section{Information Sources and Search Strategy}

This systematic review was performed in accordance with the Cochrane Collaboration guidelines and the PRISMA (Preferred Reporting Items for Systematic Reviews and Meta-Analyses) Statement. We searched scientific peer-reviewed literature published from 2000 to 2016. The controlled vocabulary (MeSh terms) and free keywords in the search strategy were defined based on the PECO question:

1. Population $(\mathrm{P})$ : pregnant women

2. Exposition (E): not applicable (determinants that influence the use of dental care and have been found during this systematic review)

3. Comparison (C): not applicable

4. The outcome $(\mathrm{O})$ : attendance to dental services

The studies were found using the electronic databases of PubMed, Scopus, Web of Science, Latin American and Caribbean Health Sciences Literature database (LILACS), Brazilian Library in 
Table 1. Electronic database and search strategy (January 9, 2017)

Medline Complete and CINAHL with full text (via EBSCO)

S9 (((S1 OR S2 OR S3 OR S4 OR S5) AND (S6 OR S7 OR S8))

S8 AB pregnancy OR AB "pregnant women” OR AB "pregnant woman" OR AB pregnant

S7 TI pregnancy OR TI "pregnant women” OR TI "pregnant woman” OR TI pregnant

S6 MH pregnancy

S5 AB "dental care" OR AB "oral health" OR AB "dental health services" OR AB “oral services" OR AB "oral health care" OR AB "dental visits" OR AB "perceived oral health" OR AB “oral health services”

S4 TI "dental care” OR TI "oral health" OR TI "dental health services” OR TI "oral services" OR TI "oral health care” OR TI "dental visits" OR TI "perceived oral health" OR TI “oral health services”

S3 MH dental health services

S2 MH dental care

S1 MH oral health

Lilacs and BBO (via Bireme)

\#1 (mh:(“pregnancy”)) OR (ab:(“gravidez”)) \#2 (ab:(“dental care”)) OR (ti:(“dental care”)) OR (mh:(“dental care”)) OR (mh:(“oral OR (ti:(“gravidez”))OR (ab:(“embarazo”))

OR (ti:(“embarazo")) health")) OR (ab:(“oral health”)) OR (ti:(“oral health”)) OR (ab:(“dental health services”)) OR (ti:(“dental health services”)) OR (mh:(“dental health services”)) OR (ab:(“dental visits”)) OR (ti:(“dental visits”)) OR (ab:(“saúde buccal”)) OR (ti:(“saúde buccal”)) OR (ab:(“atención odontológica”)) OR (ti:(“atención odontológica”)) OR (ab:(“assistência odontológica”)) OR (ti:(“assistência odontológica”)) OR (ab:(“serviços de saúde buccal”)) OR (ti:(“serviços de saúde buccal”))

$\# 1$ and \#2

PubMed

\#1pregnancy [MeSH Terms] OR pregnant women [MeSH Terms] OR "pregnant women" [Title/Abstract] OR "pregnant woman"

[Title/Abstract] OR

pregnancy [Title/Abstract] \#2oral health [MeSH Terms] OR "oral health" [Title/Abstract] OR dental care [MeSH Terms] OR dental health services [MeSH Terms] OR "dental care" [Title/Abstract] OR "dental health services" [Title/Abstract]) OR “oral services" [Title/Abstract] OR "oral health care" [Title/Abstract]) OR "dental visits" [Title/Abstract] OR "perceived oral health" [Title/Abstract] OR “oral health services” [Title/Abstract]

$\# 1$ and \#2

Web of Science

\#7 \#6 AND \#5

\#6 \#4 OR \#3

\#5 \#2 OR \#1

\#4 TI=(“dental care" OR "oral health" OR “dental health services” OR "oral services" OR "oral health care" OR "dental visits" OR "perceived oral health" OR "oral health services”)

\#3 TS=("dental care" OR "oral health" OR "dental health services" OR "oral services" OR "oral health care" OR "dental visits" OR "perceived oral health" OR “oral health services”)

\#2 TI=(pregnancy OR “pregnant women” OR “pregnant woman” OR pregnant) OR TS=(pregnancy OR "pregnant women” OR "pregnant woman” OR pregnant)

\#1 TS=(pregnancy OR “pregnant women” OR “pregnant woman” OR pregnant) OR TS=(pregnancy OR "pregnant women” OR "pregnant woman" OR pregnant)

Dentistry (BBO), Cumulative Index to Nursing and Allied Health Literature (CINAHL), and Medline (via EBSCO). A manual search was performed of the reference lists for all primary studies to obtain additional relevant publications. The related article links of each primary study in the PubMed database were also assessed.
The search strategies for all databases are included in Table 1. The strategy was appropriately adapted for each database. Full-text versions of the papers that appeared to meet the inclusion criteria (described below) were retrieved for further assessment and data extraction. 


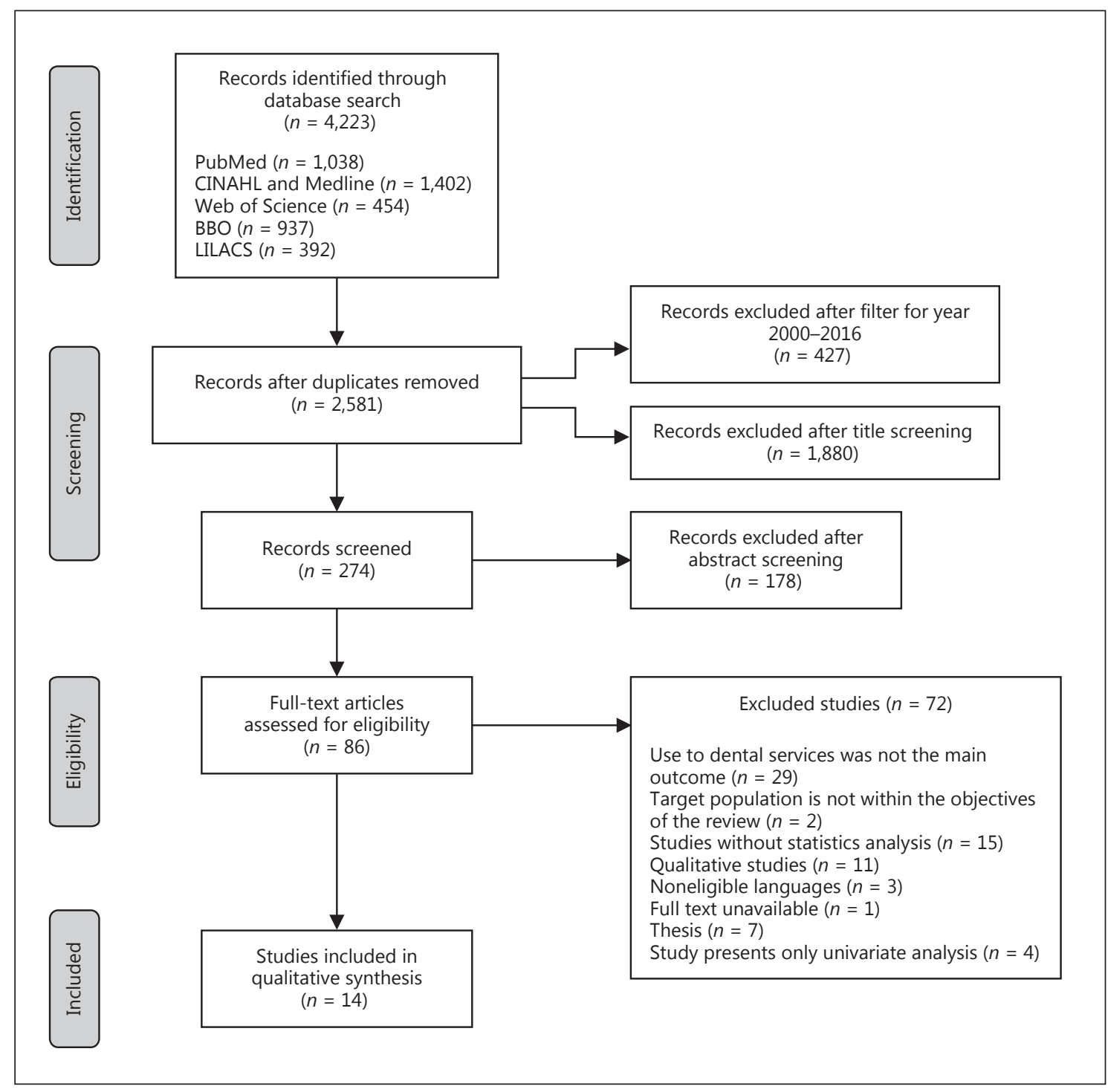

Fig. 1. Flow diagram of study identification. LILACS, Latin American and Caribbean Health Sciences Literature database; BBO, Brazilian Library in Dentistry; CINAHL, Cumulative Index to Nursing and Allied Health Literature.

\section{Eligibility Criteria}

We included observational (cross-sectional, cohort, and casecontrol) and experimental studies (randomized clinical trials) that assessed the use of dental services during pregnancy as an outcome. Studies were filtered by publication year (2000-2016) and language (English, Portuguese, Spanish, and French). Research with qualitative designs, quantitative studies with only univariate analysis or without statistical analysis, and observational studies in which the use of dental services was allocated as secondary outcome were excluded.

\section{Study Selection and Data Collection Process}

Initially, we selected the papers by title and abstract and deleted duplicate studies. Full reports were also obtained when there was insufficient information in the title and abstract to make a clear decision. Subsequently, full-text papers were acquired and 2 reviewers (J.S.R. and L.Y.A.) classified those that met the inclusion criteria. To aid in the management of a large number of studies, each eligible paper received an ID code, which combined the first author's name and the year of publication. Data were obtained by using customized extraction forms. The following information was recorded for each included study: (a) authorship and year of publication; (b) methods, including design and setting; and (c) variables related to the outcome of interest and statistical analysis.

Risk of Bias in the Assessment of Individual Studies

The studies were evaluated by 2 independent reviewers (J.S.R. and L.Y.A.) using Downs and Black's [1998] checklist for the qual- 
ity assessment of both randomized and nonrandomized studies on health care interventions. This instrument has also been recommended for assessing the risk of bias in observational designs; it consists of a checklist containing 27 items with a maximum score value of 32 points, which encompasses 5 domains: reporting, external validity, internal validity (bias), internal validity (confounding and selection bias), and power. In the present review, we employed a modified version of this instrument to assess the included studies, which were all observational. The adapted checklist consisted of 15 items (including items 1-3, 5-7, 9-11, 16, 18, 20-22, and 25 from the original list) with a maximum possible count of 15 points (higher scores indicate superior quality). During data extraction and quality assessment, disagreement between the reviewers was solved through discussion, and, if needed, consultation of a third reviewer (R.I.W.).

We also assessed risk of bias for each study according to an adapted version of the Cochrane Collaboration tool [Higgins et al., 2011], which included the 4 key domains from Downs and Black's checklist: reporting, external validity, internal validity (bias), and internal validity (confounding and selection bias). Studies were considered to have a "low" risk of bias if there was adequate sequence of reporting and clear external and internal validity information. When any criterion was not met in the reporting domain or was unable to be determined in all other key domains, a study was considered to have an "unclear" risk of bias. When one criterion was not met in the key domains, the study was considered to have a "high" risk of bias.

Summary Measures and Synthesis of the Results

We conducted data analyses with the following extracted information: author/year, title, idiom, country, study design, sample size, characteristics and source of the study population, outcome, and study findings (demographic factors, socioeconomic, psychological, and behavioral factors, and perceived need). Study characteristics and results were tabulated, and statistically significant factors were reported. Due to the heterogeneity of the outcomes and countries examined (socioeconomic and cultural differences), no meta-analysis could be undertaken. The explanatory framework for the determinants of dental service utilization during pregnancy was constructed.

\section{Results}

\section{Characteristics of the Included Papers}

After the removal of duplicates, we identified 2,581 relevant studies (Fig. 1). Subsequent to title screening, 274 papers remained; the number was then reduced to 86 after thorough examination of the abstracts. Careful reading of full texts led to the exclusion of 72 papers due to the following reasons: (1) the use of dental services was not the main outcome; (2) the target population was not within the objectives of the review; (3) a statistical analysis was not included; (4) the study was qualitative; (5) the paper contained a nonelegible language; (6) the full text was not found; and (7) it was an unpublished doctoral thesis. Four studies were excluded because they presented only univariate analysis [Oh et al., 2011; Santos Neto et al., 2012; George et al., 2013; Azofeifa et al., 2014].

Table 2 shows a summary of the information found in the final 14 articles included. All the articles presented an observational design. Four studies aimed to analyze secondary data from large demographic health surveys [Timothé et al., 2005; Marchi et al., 2010; Thompson et al., 2013; Singhal et al., 2014]; the others were primary data studies. In regard to the location, 8 studies were set in North America (7 in the United States and 1 in Canada), 2 were set in Asia (1 in Malaysia and 1 in China), 2 were set in Europe ( 1 in Greece and 1 in France), and 2 were set in Latin America (both in Colombia).

\section{Assessment of the Risk of Bias}

The assessment of the risk of bias is described in Fig. 2. Four papers [Timothé et al., 2005; Corchuelo-Ojeda, 2013; Amin and ElSalhy, 2014; Corchuelo-Ojeda and Perez, 2014] were considered to have an "unclear" risk of bias in the reporting items. Two studies [Singhal et al., 2014; Silveira et al., 2015] were considered to have a "high" risk of reporting bias. In relation to external validity, this domain was judged as "unclear" or "high" risk of bias in 7 full-text papers [Dinas et al., 2007; Boggess et al., 2010; Corchuelo Ojeda, 2013; Vergnes et al., 2013; Amin and ElSalhy, 2014; Corchuelo-Ojeda and Perez, 2014; Sun et al., 2014]. In regard to internal validity related to bias, 2 studies were considered "high" risk [Timothé et al., 2005; Dinas et al., 2007] and 3 were "unclear" [Boggess et al., 2010; Thompson et al., 2013; Silveira et al., 2015]. This was largely due to an unreliable outcome variable. Finally, for internal validity related to confounding, all studies were considered to have "low" risk of selection bias.

\section{Outcome}

For dental care attendance, we considered the outcomes "dental visits" or "tooth cleaning" as both are characterized as the use of dental services. Dental visits or dental cleaning during pregnancy were the most frequent outcome variables, and they were assessed during various stages of pregnancy: 8 studies assessed postpartum women [Al Habashneh et al., 2005; Marchi et al., 2010; Corchuelo-Ojeda, 2013; Thompson et al., 2013; Vergnes et al., 2013; Amin and ElSalhy, 2014; Corchuelo-Ojeda and Perez, 2014; Singhal et al., 2014]; 1 study included women in the third trimester [Saddki et al., 2010]; and 3 studies focused on pregnant women in all trimesters [Dinas et al., 2007; Boggess et al., 2010; Sun et al., 2014]. Two 


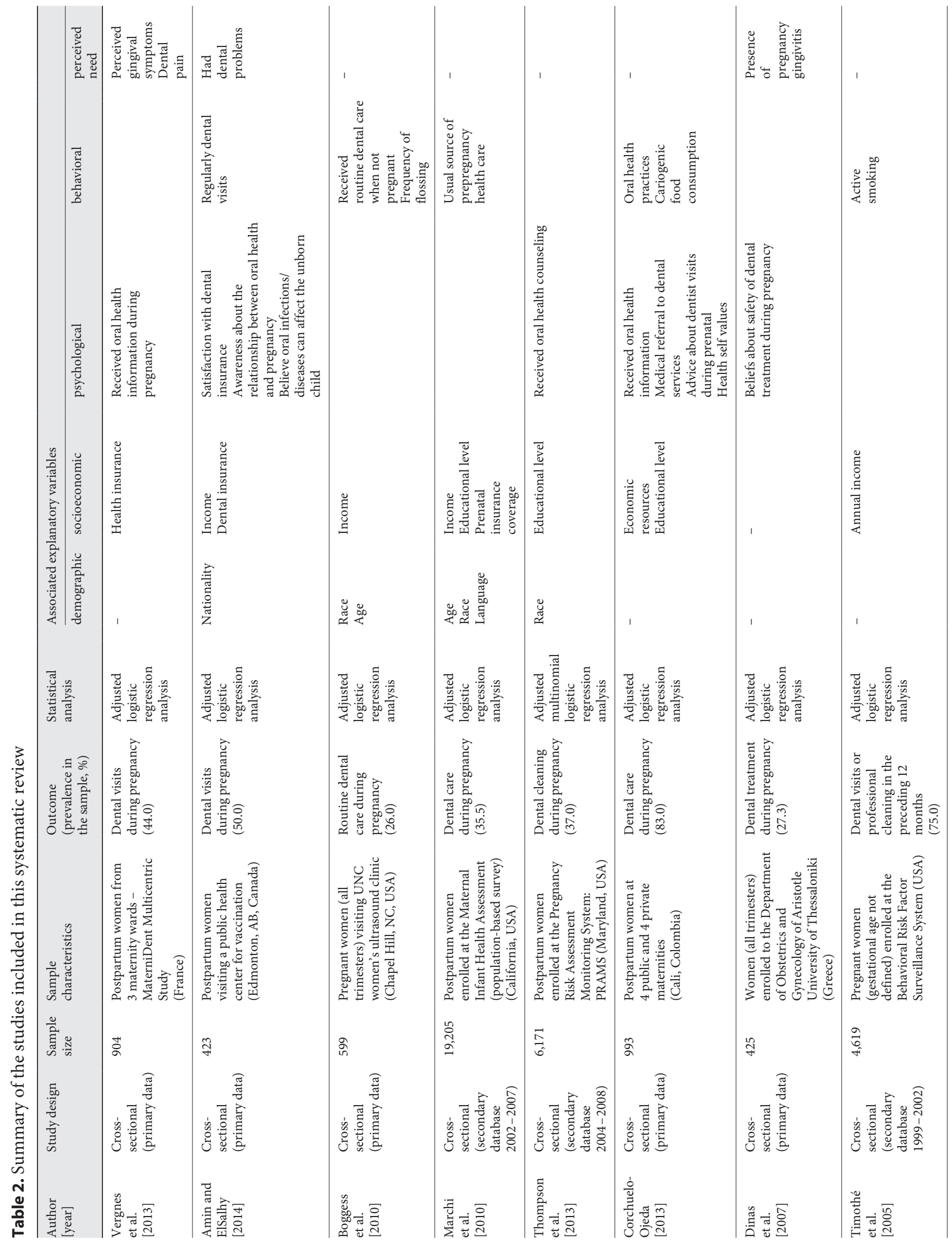




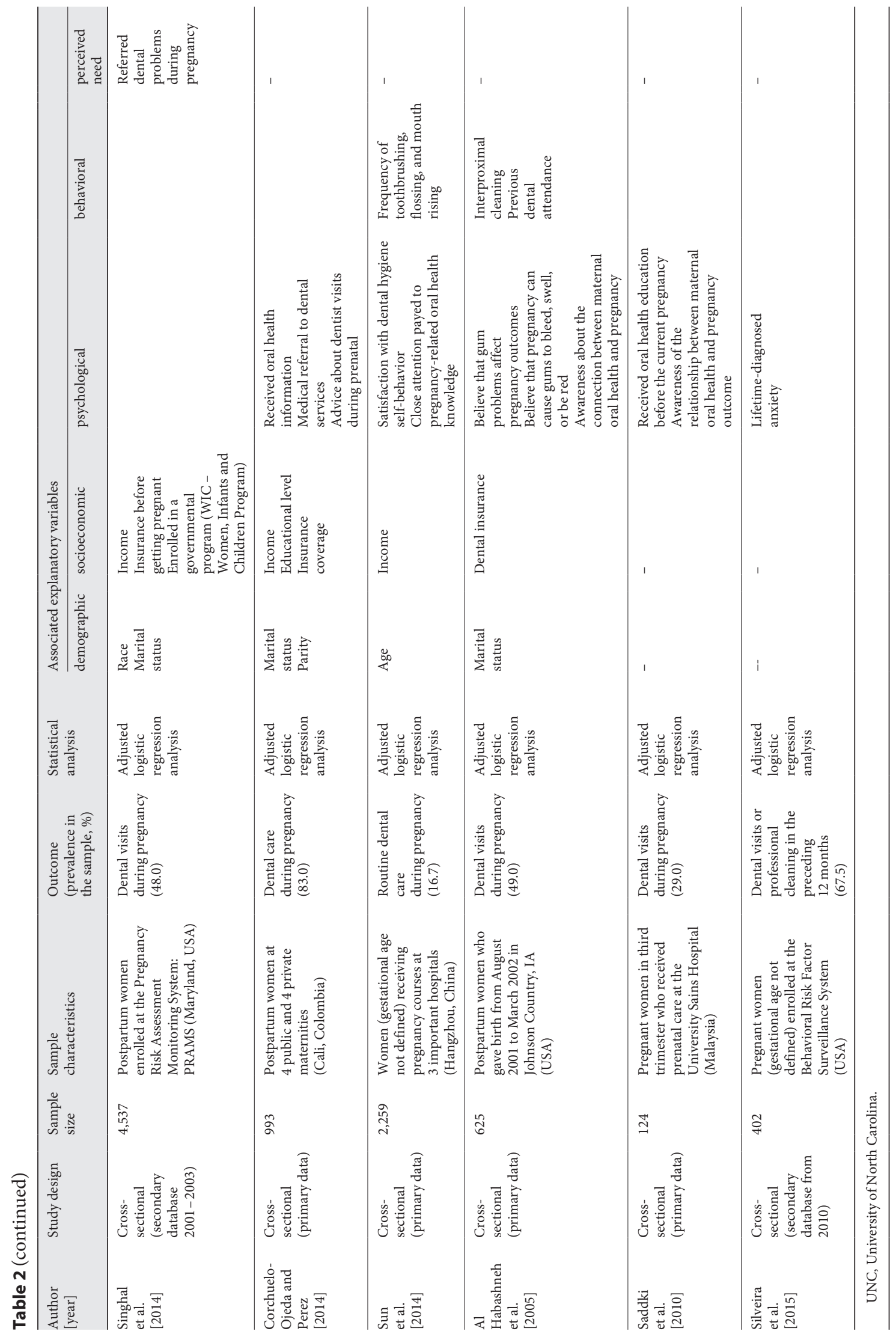


Fig. 2. Summary of quality and risk of bias assessment.

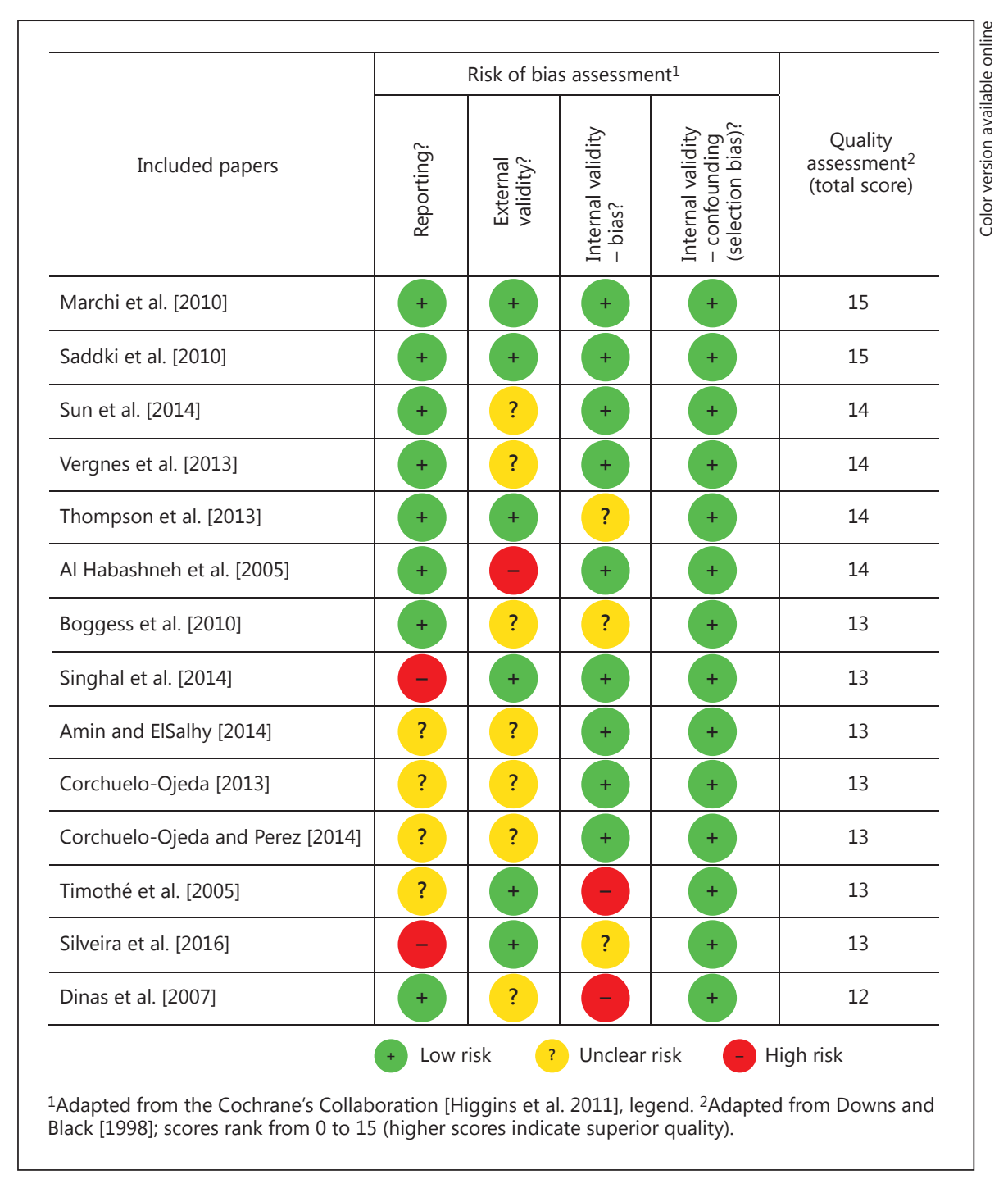

studies reported dental visits or dental cleaning in the 6 or 12 months preceding the interview regardless of the trimester of pregnancy [Timothé et al., 2005; Silveira et al., 2015].

\section{Data Synthesis}

\section{Prevalence of Dental Care Use during Pregnancy}

The use of dental services ranged from 16.7 to $83 \%$. For the studies that used routine dental care [Boggess et al., 2010; Sun et al., 2014] or dental cleaning during pregnancy [Thompson et al., 2013] as an outcome, the prevalence was between 16.7 and $37 \%$. When a visit to a dentist acted as the dependent variable, values ranged from 27.3 to
83\%; the lowest prevalence has been found in studies from Greece (27.3\%) [Dinas et al., 2007] and Malaysia (29\%) [Saddki et al., 2010]. Studies carried out in the United States and Canada showed that the prevalence of dental visits during pregnancy ranged from 33 to $67.5 \%$ [Al Habashneh et al., 2005; Marchi et al., 2010; Amin and ElSalhy, 2014; Singhal et al., 2014; Silveira et al., 2015]. Similar results were found in France, with a prevalence of $56 \%$ [Vergnes et al., 2013]. The highest prevalence (83\%) was described in a cross-sectional study performed in Cali, Colombia [Corchuelo-Ojeda, 2013; Corchuelo-Oje$\mathrm{da}$ and Perez, 2014]. Considering the utilization of dental services in the previous year as the outcome, the prevalence was around 70\% [Timothé et al., 2005; Silveira et al., 2015]. 


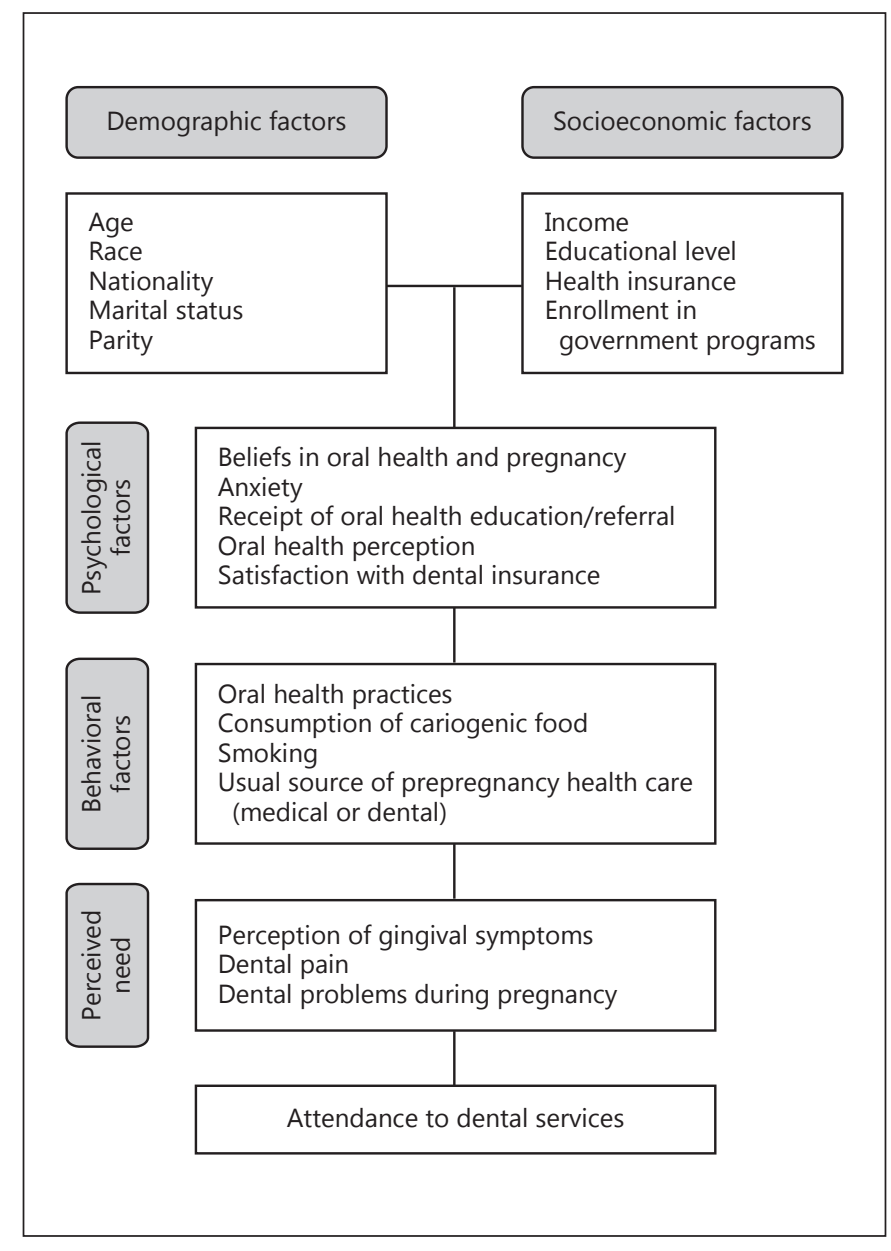

Fig. 3. Explanatory framework based on the systematic review.

Determinants of Dental Care Use during Pregnancy

The final explanatory framework is described in Figure 3. The determinants were classified as demographic, socioeconomic, psychological, and behavioral factors, and perceived need.

\section{Demographic Factors}

In this review, demographic factors included women's age, marital status, parity, and ethnicity/nationality/language. Three studies have found that dental care utilization was associated with age. In a study conducted in Chapel Hill, NC, USA, the percentage of women aged 36 years or older who had routine dental care utilization during pregnancy was lower than in its younger counterpart [Boggess et al., 2010]. This result was in contrast to what was obtained from a study in China [Sun et al., 2014]. Additionally, in a large secondary data study encompassing a representative sample from California, USA, pregnant women who had not received dental care during pregnancy were primarily younger [Marchi et al., 2010].

Some papers indicated that married women were more likely to look for dental care than single or unmarried ones [Al Habashneh et al., 2005; Corchuelo-Ojeda 2013; Singhal et al., 2014]. The relationship between parity and dental service utilization was investigated in 5 papers [Dinas et al., 2007; Marchi et al., 2010; Saddki et al., 2010; Vergnes et al., 2013; Corchuelo-Ojeda and Perez, 2014], but only in the Colombian study [Corchuelo-Ojeda, 2013] did first-time mothers have significantly more dental visits than pregnant women with 2 or more children.

Five studies had ethnicity, nationality, or language associated with dental care utilization in their multivariate explanatory models [Boggess et al., 2010; Marchi et al., 2010; Thompson et al., 2013; Amin and ElSalhy, 2014; Singhal et al., 2014]. In Canada, Amin and ElSalhy [2014] found that Canadian-born women were $48 \%$ more likely to have visited the dentist during pregnancy than nonCanadian ones. In the Californian survey, Marchi et al. [2010] identified that the odds of not receiving dental care during pregnancy were higher among ethnic minorities and those who spoke non-English languages at home. In one study from Maryland (USA), black women had lower odds of receiving dental cleaning during pregnancy than the white ones [Thompson et al., 2013]. Similarly, Singhal et al. [2014] demonstrated that nonwhite pregnant women had lower odds of visiting a dentist than white ones.

\section{Socioeconomic Factors}

Income, educational level, and health insurance were the socioeconomic factors related to the use of dental care during pregnancy. Income was a significant predictor for not reporting dental service utilization in the previous 12 months [Timothé et al., 2005] or during pregnancy [Boggess et al., 2010; Marchi et al., 2010; Amin and ElSalhy, 2014; Corchuelo-Ojeda and Perez, 2014; Singhal et al., 2014]. Low-income pregnant women were less likely to have a routine dental care than high-income ones [Sun et al., 2014]. Two studies did not show a statistically significant association between dental service utilization during pregnancy and income [Al Habashneh et al., 2005; Saddki et al., 2010].

Educational level was found to be a significant factor for the utilization of dental services in adjusted models [Corchuelo-Ojeda, 2013; Thompson et al., 2013; Corchuelo-Ojeda and Perez, 2014].Women who had at least 12 years of schooling had significantly higher odds of receiving dental cleaning during pregnancy [Thompson et al., 
2013]. However, several studies suggested that women's educational level was not a significant predictor of dental service utilization during pregnancy after multivariate analysis [Al Habashneh et al., 2005;Timothé et al., 2005; Boggess et al., 2010; Marchi et al., 2010; Saddki et al., 2010; Vergnes et al., 2013; Amin and ElSalhy, 2014; Singhal et al., 2014; Sun et al., 2014].

In 4 studies [Al Habashneh et al., 2005; Vergnes et al., 2013; Amin and ElSalhy, 2014; Corchuelo-Ojeda and Perez, 2014], health insurance coverage was strongly associated with dental visits during pregnancy. Women who had health insurance before getting pregnant were more likely to visit a dentist than women who were not insured [Singhal et al., 2014]. Similarly, Marchi et al. [2010] found that the odds of not receiving dental care during pregnancy were higher among women who lacked first-trimester insurance coverage.

Besides insurance, the enrollment in governmental programs was also a predictor of dental service utilization. Pregnant women enrolled in a US-based program that targets low-income women, infants, and children under 5 who are at nutrition risk were more likely to have visited a dentist than women who were not insured and did not enroll in the program [Singhal et al., 2014].

\section{Psychological Factors}

Many psychological factors have been associated with the utilization of dental care: receipt of oral health education/referral, oral health perception, beliefs in oral health and pregnancy, and anxiety and satisfaction with dental insurance. In regard to oral health education, 6 studies have analyzed this variable [Saddki et al., 2010; Corchuelo-Ojeda, 2013; Thompson et al., 2013; Vergnes et al., 2013; Corchuelo-Ojeda and Perez, 2014; Singhal et al., 2014], and only 1 did not find any statistical association after adjusting for confounding factors [Singhal et al., 2014]. Pregnant women who had high levels of knowledge related to oral health were more likely to have visited the dentist than those with poor knowledge [Corchuelo-Ojeda, 2013]. Receipt of dental health education was also significantly associated with the use of dental services during pregnancy. In the study by Corchuelo-Ojeda and Perez [2014], women who received prenatal oral health counseling were more likely to seek dental services. Similar results were described in 3 other studies [Saddki et al., 2010; Thompson et al., 2013; Vergnes et al., 2013]. Pregnant women who received medical referrals or advice about dental visits were more likely to have used dental services during pregnancy [Corchuelo-Ojeda, 2013; Corchuelo-Ojeda and Perez, 2014]. Regarding oral health perception, pregnant women who rated their oral health as good went to the dentist more often than those who considered it regular or poor [Corchuelo-Ojeda, 2013].

Five studies examined the association between the use of dental services and oral health and pregnancy-related beliefs. The recognition of the relationship between maternal oral health and pregnancy outcomes, and the belief that the mother's oral infection can affect the unborn child was significantly associated with dental care attendance [Saddki et al., 2010; Amin and ElSalhy, 2014]. On the other hand, the perception that dental treatment during pregnancy is unsafe was an important factor in limiting the utilization of dental care [Dinas et al., 2007]. In the study by Al Habashneh et al. [2005], pregnant women who answered positively to the questions "Have you heard about the connection between oral health and pregnancy?" and "Do you think tooth and gum problems could affect outcomes of pregnancy?" were significantly more likely to report a dental visit during pregnancy.

The association between dental care utilization and anxiety was tested in a sample of pregnant women [Silveira et al., 2015]. After adjusting for socioeconomic factors, respondents with lifetime-diagnosed anxiety had increased odds of reporting no dental care utilization in the previous year compared to those without the disorder.

\section{Behavioral Factors}

Oral health practices, cariogenic food consumption, smoking, and usual source of prepregnancy care are all identified behavioral factors.

Oral health practices were associated with dental care attendance. Pregnant women with good hygiene had higher odds of using dental services and receiving routine dental care than those with poor hygiene [CorchueloOjeda, 2013]. Boggess et al. [2010] identified that women with infrequent flossing were less likely to seek routine dental care during pregnancy. Al Habashneh et al. [2005] found similar results for interproximal dental cleaning. Frequency of toothbrushing, mouth rinsing, and flossing were significant predictors of dental care attendance in a Chinese study [Sun et al., 2014], as was the self-perceived satisfaction with one's own dental hygiene behavior.

Likewise, pregnant women who did not consume cariogenic foods [Corchuelo-Ojeda, 2013] and did not smoke [Timothé et al., 2005] were significantly more likely to use dental services.

Having a usual source of prepregnancy health care, either medical [Marchi et al., 2010] or dental [Al Habashneh et al., 2005; Amin and ElSalhy, 2014], was positively associated with dental visits for pregnant women. Fre- 
quency of dental visits when not pregnant and having a regular source of prepregnancy medical service were strongly associated with increased utilization of dental services during pregnancy [Marchi et al., 2010; Amin and ElSalhy, 2014]. Al Habashneh et al. [2005] found that mothers reporting dental visits every 6 months to 1 year when not pregnant were more likely to report dental visits during pregnancy compared to those who reported visits every 2 years when not pregnant.

\section{Perceived Need}

Four studies found a relationship between perceived need and dental visits during pregnancy. Women with referred symptoms of gingivitis [Dinas et al., 2007; Vergnes et al., 2013], dental pain [Vergnes et al., 2013], or perceived dental problems [Amin and ElSalhy, 2014; Singhal et al., 2014] were more likely to use dental services.

\section{Discussion}

Understanding the determinants of health care use by vulnerable populations, such as pregnant women, is important for the organization of services, allocation of resources, definition of action strategies, and indicators for impact assessment [Andersen and Davidson, 1997; US Department of Health and Human Services, 2000; National Research Council, 2012]. Some studies have shown differences in self-reported oral health conditions and dental visits between pregnant and nonpregnant women. Differences in reported use of dental care may be related to delayed postpartum dental care due to the myths and beliefs of pregnant women and the insecurity of health professionals to avoid potential adverse pregnancy outcomes [Al Habashneh et al., 2005; Timothé et al., 2005; Azofeifa et al., 2014].

It is well defined that oral health should integrate health care for pregnant women and their newborns. This review identified the factors related to the utilization of dental services during pregnancy, which were organized in a hierarchical explanatory framework. It is important to increase knowledge about the determinants that predict dental service utilization during pregnancy; it could lead to policy strategies that go far beyond oral health education and help to improve women's access to adequate dental services [Marchi et al., 2010; Vergnes et al., 2013].

Studies with dental service utilization as a secondary outcome and those using solely univariate analyses were excluded because the sample size is always calculated

Determinants of Dental Care Attendance during Pregnancy based on the primary outcome, and it is not necessarily significant to the secondary outcome [Schulz et al., 2010]. According to the evaluation instrument for the quality of the articles, studies that did not control confounding factors had low scores and thus low quality [Downs and Black, 1998]. As our goal was to synthesize the best evidence, we decided to exclude such studies from synthesis.

Despite the low number of eligible papers, this review summarizes relevant information. Of the 14 studies included, 2 reached the highest level of quality and lowest risk of bias [Marchi et al., 2010; Saddki et al., 2010]. They indicate significant evidence for the relationship between dental care utilization during pregnancy and demographic, socioeconomic, psychological, and behavioral determinants. Four studies assessed perceived need. Although one of them reached low scores in the overall analysis [Dinas et al., 2007], the others were classified as low risk of bias [Vergnes et al., 2013; Amin and ElSalhy, 2014; Singhal et al., 2014] in regard to internal validity assessment (bias and confounding levels), which reinforces the odds of a positive relationship between dental care attendance and referred dental problems (including pain) during pregnancy.

Four studies were performed with secondary databases from US official systems and population-based surveys [Marchi et al., 2010; Thompson et al., 2013; Singhal et al., 2014; Silveira et al., 2015]. They have a well-defined and standardized sampling methodology, which led to classifying them as high external validity. Nevertheless, 8 studies included in this review did not discuss the representativeness of the data, resulting in questionable external validity and thus not allowing to generalize the results to the overall population.

The main issue regarding the risk of internal validity (bias) was the chosen outcome. Five studies adopted dependent variables that were considered nonreliable to the object of this review [Timothé et al., 2005; Dinas et al., 2007; Boggess et al., 2010; Sun et al., 2014; Silveira et al., 2015]. For example, dental visits or dental cleaning in the preceding 6 months to 1 year [Timothé et al., 2005; Silveira et al., 2015] may not reflect pregnancy status at the time of dental attendance.

The use of evidence-based conceptual frameworks to guide the objectives and research design is important, as they assist in the interpretation of statistical outcomes in multivariate analysis [Holst et al., 2001; Ricketts and Goldsmith, 2005]. We summarized the results of the present review in a hierarchical framework by classifying the determinants of dental service utilization during preg- 
nancy in 5 categories: demographic, socioeconomic, psychological, behavioral, and perceived need. Disregarding the existence of some theory-based frameworks that intend to explain the use of health services [Aday and Andersen, 1974], almost all the included studies did not have their analyses referred by conceptual explanatory models as a rationale to select the independent variables [Heider et al., 2014; Lowe et al., 2016].

According to the present review, among others, the low uptake of dental care during pregnancy can be attributed to demographic and socioeconomic factors. The relationship between age and use of services is not clear. Most studies did not find a statistical association, and those with a significant relationship displayed conflicting results. This finding could be related to the difference in the cutoff point of the age variable among the studies, and we recommend standardization for further research.

Ethnicity, nationality/language, and educational level were also important, as white women [Thompson et al., 2013; Singhal et al., 2014] and those who were born in the country where the study took place [Amin and ElSalhy, 2014] were more likely to visit the dentist during pregnancy. Likewise, higher odds were found among those with higher educational levels [Corchuelo-Ojeda, 2013; Thompson et al., 2013; Corchuelo-Ojeda and Perez, 2014], which may be related to higher income. Minority and low-income women may not seek dental care during pregnancy because of stressors, such as poor domestic relationships and personal finances, perception of dental experience, attitudes toward dental providers, importance attributed to oral health, time constraints, and additional factors [Le et al., 2009].

The role of health insurance was assessed by comparing studies from different countries. In Cali, Colombia [Corchuelo-Ojeda, 2013], 83\% of the pregnant women reported dental visits; however, only $11.5 \%$ of them did not have insurance. The review identified that countries with some type of public warranty of access for low-income pregnant women also had higher proportions of dental service utilization [Thompson et al., 2013; Singhal et al., 2014] with rates of $50 \%$ or higher. However, it seems that the mere offer of dental services does not guarantee adequate prenatal dental care [Santos Neto et al., 2012].

Oral health education and access to good-quality information also appear to be important behavioral factors, which enable the use of dental services during pregnancy. The studies indicated that pregnant women should be informed about the importance of maintaining good oral health during pregnancy and the relationship between maternal oral health status and future health of their children [Oh et al., 2011]; the studies also point out that efforts should be made to overcome barriers, such as beliefs [Dinas et al., 2007]. They indicate that pregnant stressors, low priority given to dental care, safety misconceptions, and the belief that dental care during the first 3 months of pregnancy is harmful to the baby are important factors that may affect the use of dental services during pregnancy [Le et al., 2009; Detman et al., 2010; Nogueira et al., 2012].

Perceived need has usually been associated with dental service utilization. Despite the high report of dental problems during pregnancy in some studies, the proportion of women who sought dental care was low [Singhal et al., 2014]. In the study by Dinas et al. [2007], only 27.3 of $48.8 \%$ pregnant women who reported symptoms of gingivitis visited the dentist.

Finally, some methodological issues need to be taken into account when interpreting these findings. First, all the studies included have cross-sectional designs, which does not allow for inferring causality [Mann, 2003]. Second, the interpretation of the evidence level is also limited by the low amount of published literature on factors associated with the utilization of dental services during pregnancy. For example, we found few quantitative studies that focus on psychological influences such as beliefs, myths, and dental anxiety, which seems to be increased during pregnancy [Le et al., 2009; Detman et al., 2010; Nogueira et al., 2012]. Third, some poor-quality studies were found, and they also vary widely in terms of content, sample size, and outcome. This heterogeneity made it difficult to interpret and summarize key findings and to explore similarities worldwide as most reviewed studies were conducted in the United States and Canada, which reduces the external validity of the findings of this review.

Besides the limitations, our findings highlight the factors that enable or restrict the utilization of dental services for pregnant women and should guide policymakers to breaking barriers for this vulnerable group; this not only includes the socioeconomic barriers but also behavioral and psychological barriers. Investment in health education and prevention should be among the priorities as pregnancy is a period in life when women face many fears and insecurities, and some health professionals do not feel comfortable referring them to dental treatment. Finally, we suggest that well-designed studies addressing psychosocial factors are still necessary to better understand the use of dental services by pregnant women. 


\section{Conclusion}

Demographic, socioeconomic, psychological, and behavioral factors, and perceived need were identified as determinants of dental service utilization during pregnancy. Well-designed studies with reliable outcomes are required to confirm the findings described in this review and to increase the strength of the evidence.

\section{Disclosure Statement}

The authors have no conflicts of interest to declare.

\section{Author Contributions}

J.S.R. contributed to conception and design, acquisition of the study, analysis and interpretation of data and drafted the manuscript; L.Y.A. contributed to data acquisition and analysis. R.I.W. contributed to the study design and analysis of the data; S.J.M. contributed to conception of the study and interpretation of the data; M.H.B. contributed to conception and design of the study and interpretation of the data. All authors critically revised the manuscript, had final responsibility for the decision to submit, and approved the submitted version.

\section{References}

Aday LA: Health status of vulnerable populations. Annu Rev Public Health 1994;15:487-509.

Aday LA, Andersen R: A framework for the study of access to medical care. BMC Health Serv Res 1974;9:208.

Al Habashneh R, Guthmiller JM, Levy S, Johnson GK, Squier C, Dawson DV, Fang Q: Factors related to utilization of dental services during pregnancy. J Clin Periodontol 2005;32:815-821.

Albuquerque OMRd, Abegg C, Rodrigues CS: Pregnant women's perceptions of the Family Health Program concerning barriers to dental care in Pernambuco, Brazil (in Portuguese). Cad Saude Publica 2004;20:789-796.

Amin M, ElSalhy M: Factors affecting utilization of dental services during pregnancy. J Periodontol 2014;85:1712-1721.

Andersen R: A Behavioral Model of Families' Use of Health Services. Res Ser 25. Chicago, Center for Health Administration Studies, University of Chicago, 1968.

Andersen R, Davidson P: Ethnicity, aging, and oral health outcomes: a conceptual framework. Adv Dent Res 1997;11:203-209.

Anderson C, Harris MS, Kovarik R, Skelton J: Discovering expectant mothers' beliefs about oral health: an application of the Centering Pregnancy Smiles Program. Int Q Community Health Educ 2009-2010;30:115-140.

Araújo CS, Lima Rda C, Peres MA, Barros AJD: Use of dental services and associated factors: a population-based study in southern Brazil (in Portuguese). Cad Saude Publica 2009;25: 1063-1072.

Azofeifa A, Yeung LF, Alverson CJ, Beltran-Aguilar E: Oral health conditions and dental visits among pregnant and nonpregnant women of childbearing age in the United States, National Health and Nutrition Examination Survey, 1999-2004. Prev Chronic Dis 2014;11:E163.

Baker SR: Applying Andersen's behavioural model to oral health: what are the contextual factors shaping perceived oral health outcomes? Community Dent Oral Epidemiol 2009;37: 485-494.
Boggess KA, Urlaub DM, Massey KE, Moos MK, Matheson MB, Lorenz C: Oral hygiene practices and dental service utilization among pregnant women. J Am Dent Assoc 2010;141: 553-561.

Codato LA, Nakama L, Melchior R: The beliefs of pregnant women about dental care during gestation (in Portuguese). Cien Saude Colet 2008; 13:1075-1080.

Corbella S, Taschieri S, Del Fabbro M, Francetti L, Weinstein R, Ferrazzi E: Adverse pregnancy outcomes and periodontitis: a systematic review and meta-analysis exploring potential association. Quintessence Int 2016;47:193204.

Corchuelo-Ojeda J: Social determinants and lifestyle on oral health in access to dental caleñas pregnant in 2012 (in Spanish). Rev Fac Nac Salud Pública 2013;31:170-180.

Corchuelo-Ojeda J, Perez GJ: Socioeconomic determinants of dental care during pregnancy in Cali, Colombia (in Spanish). Cad Saude Publica 2014;30:2209-2218.

Detman LA, Cottrell BH, Denis-Luque MF: Exploring dental care misconceptions and barriers in pregnancy. Birth 2010;37:318-324.

Dinas K, Achyropoulos V, Hatzipantelis E, Mavromatidis G, Zepiridis L, Theodoridis T, Dovas D, Tantanasis T, Goutzioulis F, Bontis $\mathrm{J}$ : Pregnancy and oral health: utilisation of dental services during pregnancy in northern Greece. Acta Obstet Gynecol Scand 2007;86: 938-944.

Downs SH, Black N: The feasibility of creating a checklist for the assessment of the methodological quality both of randomised and nonrandomised studies of health care interventions. J Epidemiol Community Health 1998; 52:377-384.

Figuero E, Carrillo-de-Albornoz A, Martín C, Tobías A, Herrera D: Effect of pregnancy on gingival inflammation in systemically healthy women: a systematic review. J Clin Periodontol 2013;40:457-473.
George A, Johnson M, Blinkhorn A, Ajwani S, Bhole S, Yeo AE, Ellis S: The oral health status, practices and knowledge of pregnant women in south-western Sydney. Aust Dent J 2013; 58:26-33.

Hausmann-Muela S, Ribera JM, Nyamongo I: Health-seeking behaviour and the health system response. Disease Control Priorities Project Working Paper 14. 2003, https://pdfs.semanticscholar.org/3511/6a3c0a88898a531ce 214e4448861ae00e320.pdf.

Heider D, Matschinger H, Müller H, Saum K-U, Quinzler R, Haefeli WE, Wild B, Lehnert T, Brenner H, König H-H: Health care costs in the elderly in Germany: an analysis applying Andersen's behavioral model of health care utilization. BMC Health Serv Res 2014;14:1.

Higgins JP, Altman DG, Gøtzsche PC, Jüni P, Moher D, Oxman AD, Savović J, Schulz KF, Weeks L, Sterne JA: The Cochrane Collaboration's tool for assessing risk of bias in randomised trials. BMJ 2011;343:d5928.

Hill K, Chadwick B, Freeman R, O'Sullivan I, Murray J: Adult Dental Health Survey 2009: relationships between dental attendance patterns, oral health behaviour and the current barriers to dental care. Br Dent J 2013;214: 25-32.

Holst D, Schuller AA, Aleksejuniené J, Eriksen HM: Caries in populations - a theoretical, causal approach. Eur J Oral Sci 2001;109:143148 .

Ide M, Papapanou PN: Epidemiology of association between maternal periodontal disease and adverse pregnancy outcomes - systematic review. J Clin Periodontol 2013;40(suppl 14):S181-S194.

Jevtić M, Pantelinac J, Jovanović-Ilić T, Petrović $\mathrm{V}$, Grgić $\mathrm{O}$, Blažić L: The role of nutrition in caries prevention and maintenance of oral health during pregnancy. Med Pregl 2015;68: 387-393.

Keirse MJN, Plutzer K: Women's attitudes to and perceptions of oral health and dental care during pregnancy. J Perinat Med 2010;38:3-8. 
Kumar PS: From focal sepsis to periodontal medicine: a century of exploring the role of the oral microbiome in systemic disease. J Physiol 2017;595:465-476..

Le M, Riedy C, Weinstein P, Milgrom P: Barriers to utilization of dental services during pregnancy: a qualitative analysis. J Dent Child 2009;76:46-52.

Locker D, Maggirias J, Quiñonez C: Income, dental insurance coverage, and financial barriers to dental care among Canadian adults. J Public Health Dent 2011;71:327-334.

Lowe SR, Norris FH, Galea S: Mental health service utilization among natural disaster survivors with perceived need for services. Psychiatr Serv 2016;67:354-357.

Lu H-X, Xu W, Wong MCM, Wei T-Y, Feng X-P: Impact of periodontal conditions on the quality of life of pregnant women: a cross-sectional study. Health Qual Life Outcomes 2015;13: 67-67.

Mann C: Observational research methods. Research design II: cohort, cross sectional, and case-control studies. Emerg Med J 2003;20: 54-60.

Marchi KS, Fisher-Owen SA, Weintraub JA, Yu $\mathrm{Z}$, Braveman PA: Most pregnant women in California do not receive dental care: findings from a population-based study. Public Health Rep 2010;125:831-842.

Marshman Z, Porritt J, Dyer T, Wyborn C, Godson J, Baker S: What influences the use of dental services by adults in the UK? Community Dent Oral Epidemiol 2012;40:306-314.

National Research Council: Improving Access to Oral Health Care for Vulnerable and Underserved Populations. Washington, National Academies Press, 2012.

NHS UK: What are my rights during pregnancy? 2016, www.Nhs.Uk/chq/pages/953.Aspx? Categoryid $=54 \&$.

Nogueira LT, Valsecki Júnior A, Martins CR, Rosell FL, Silva SRCd: Delay in seeking dental treatment and perception of oral health in pregnant women. Odontol Clín-Cient 2012; $11: 127-131$

Oh J, Leonard L, Fuller D, Miller K: Less than optimal oral health care during pregnancy in Rhode Island women: oral health care as a part of prenatal care. Med Health RI 2011;94: 141-143.
Oliveira BH, Nadanovsky P: The impact of oral pain on quality of life during pregnancy in low-income Brazilian women. J Orofac Pain 2006;20:297-305.

Petersen PE: World health organization global policy for improvement of oral health World Health Assembly 2007. Int Dent J 2008; $58: 115-121$.

Pinto RS, Matos DL, de Loyola Filho AI: Characteristics associated with the use of dental services by the adult Brazilian population (in Portuguese). Cien Saude Col 2012;17:531544.

Pohjola V, Lahti S, Vehkalahti MM, Tolvanen M, Hausen H: Association between dental fear and dental attendance among adults in Finland. Acta Odontol Scand 2007;65:224-230.

Radnai M, Gorzó I, Nagy E, Urbán E, Eller J, Novák T, Pál A: The oral health status of postpartum mothers in south-east Hungary. Community Dent Health 2007;24:111-116.

Reisine S: A path analysis of the utilization of dental services. Community Dent Oral Epidemiol 1987;15:119-124

Ricketts TC, Goldsmith LJ: Access in health services research: the battle of the frameworks. Nurs Outlook 2005;53:274-280.

Rosenstock IM: Why people use health services. Milbank Mem Fund Q 1966;44(suppl):94127.

Saddki N, Yusoff A, Hwang YL: Factors associated with dental visit and barriers to utilisation of oral health care services in a sample of antenatal mothers in Hospital Universiti Sains Malaysia. BMC Public Health 2010;10:75.

Santos Neto ETd, Oliveira AE, Zandonade E, Leal MdC: Access to dental care during prenatal assistance (in Portuguese). Ciênc Saúde Colet 2012;17:3057-3068.

Scheutz F, Heidmann J: Determinants of utilization of dental services among 20- to 34-yearold Danes. Acta Odontol Scand 2001;59:201208.

Schulz KF, Altman DG, Moher D; CONSORT Group: CONSORT 2010 Statement: updated guidelines for reporting parallel group randomised trials. BMC Medicine 2010;8:18

Shanthi V, Vanka A, Bhambal A, Saxena V, Saxena S, Kumar SS: Association of pregnant women periodontal status to preterm and low-birth weight babies: a systematic and evidence-based review. Dent Res J 2012;9:368380.
Shaw C, Brittain K, Tansey R, Williams K: How people decide to seek health care: a qualitative study. Int J Nurs Stud 2008;45:1516-1524.

Silveira ML, Whitcomb BW, Pekow P, Carbone ET, Chasan-Taber L: Anxiety, depression, and oral health among US pregnant women: 2010 Behavioral Risk Factor Surveillance System. J Public Health Dent 2016;76:56-64.

Singhal A, Chattopadhyay A, Garcia AI, Adams $A B$, Cheng D: Disparities in unmet dental need and dental care received by pregnant women in Maryland. Matern Child Health J 2014;18:1658-1666.

Slack-Smith L, Mills C, Bulsara MK, O’Grady M Demographic, health and lifestyle factors associated with dental service attendance by young adults. Aust Dent J 2007;52:205-209.

Sun W, Guo J, Li XY, Zhao YQ, Chen H, Wu G: The routine utilization of dental care during pregnancy in eastern China and the key underlying factors: a Hangzhou city study. PLoS One 2014;9:e98780.

Thompson TA, Cheng D, Strobino D: Dental cleaning before and during pregnancy among Maryland mothers. Matern Child Health J 2013;17:110-118.

Timothé P, Eke PI, Presson SM, Malvitz DM: Dental care use among pregnant women in the United States reported in 1999 and 2002. Prev Chronic Dis 2005;2:A10.

US Department of Health and Human Services: Healthy People 2010. With Understanding and Improving Health and Objectives for Improving Health. Washington, US Government Printing Office, 2000, vol 2.

Vergnes JN, Pastor-Harper D, Constantin D, Bedos C, Kaminski M, Nabet C, Sixou M, Rouillon F: Perceived oral health and use of dental services during pregnancy: the MaterniDent study (in French). Sante Publique 2013;25: 281-292.

Woods MD, Kirk MD, Agarwal MS, Annandale E, Arthur T, Harvey J, Hsu R, Katbamna S, Olsen R, Smith L: Vulnerable groups and access to health care: a critical interpretive review. National Coordinating Centre NHS Service Delivery Organ R D (NCCSDO) 2005; 27:2012. 